\title{
Interpolation-based height analysis for improving a recurrence solver
}

\author{
Manuel Montenegro ${ }^{1}$, Olha Shkaravska², \\ Marko van Eekelen ${ }^{2 \& 3}$, and Ricardo Peña ${ }^{1}$ \\ ${ }^{1}$ Universidad Complutense Madrid \\ 2 Radboud University Nijmegen \\ ${ }^{3}$ Open University of the Netherlands \\ \{montenegro\}@fdi.ucm.es, \{shkarav,marko\}@cs.ru.nl,\{ricardo@sip.ucm.es\}
}

\begin{abstract}
The COSTA system infers resource consumption bounds from Java bytecode using an internal recurrence solver PUBS. This paper suggests an improvement of the COSTA system, such that it can solve a larger number of recurrences. The idea is to replace one of its static analyses, the ranking function analysis, by another kind of analysis, height analysis, in such a way that polynomial bounds of any degree may be inferred instead of just linear expressions. The work can be seen as an application of some polynomial interpolation techniques used by some of the authors in prior analyses. Finding a way to choose proper test nodes is the key to the solution presented in this paper.
\end{abstract}

\section{Introduction}

The application of resource analysis techniques to actual programs frequently leads to generating cost recurrence equations which must be solved, i.e. expressed in a closed form in order to be useful. There have been many attempts to solve this kind of equations in an automatic way. Most of the tools doing this are restricted to some specific recurrence families, frequently on only one variable.

A system proved successful in generating and solving multivariate recurrence equations is COSTA [1], together with its associated recurrence solving subsystem PUBS [2]. COSTA is given a program text written in Java bytecode, and the kind of resource to be analysed (number of instructions executed, memory consumption, and others), and in many cases it is able to obtain symbolic upper bounds for these runtime figures. The bounds are expressed as multivariate functions on the sizes of the input arguments of the method being analysed.

Internally, the system contains many different static analyses which we briefly summarise in Sec. 2. In this paper, we focus on one of them: the ranking function synthesis done in one of the steps. Its aim is to obtain an upper bound on the number of unfoldings the recurrence equations must undergone to reach

This work was partly funded by the EU Artemis Joint Undertaking in the CHARTER project, grant-nr. 100039, and by Spanish FPU grant AP2006-02154 and projects TIN2008-06622-C03-01 (STAMP), S2009/TIC-1465 (PROMETIDOS). 
a base case, i.e. to reach a case with does not admit more unfoldings. This number corresponds with the maximum height of the so-called evaluation trees. As we will see, this ranking function is crucial in the computation of the resource upper bound. The limitation of this step is that the ranking function must be a linear expression on the argument sizes. The Podelski and Rybalchenko's method [19], which is known to be complete for linear ranking functions, is used to this purpose.

In the past, we have used polynomial interpolation techniques in different types of size analysis [20], and also in synthesising polynomial ranking functions for Java loops [22]. The main idea there was to synthesise polynomials by evaluating program fragments, or particular term rewriting systems, in some specific points forming a so-called NCA configuration (Node Configuration A, [7]). By adopting this configuration the number of test points needed is a precise function of the polynomial degree and the number of variables.

We apply the same idea here by evaluating recurrence equations in well chosen points and then interpolating a multivariate polynomial of known degree passing through those points. As we are only interested in the maximum height of the recurrence evaluation trees (the rest of the recurrence solving process is already done by PUBS), we first write a derived recurrence defining this height. The obtained polynomial would be then an upper bound of the height of any evaluation tree. The degree must be guessed, as it happens in other techniques inferring arbitrary polynomials $[17,13]$, and additionally the interpolated polynomial must be proved correct w.r.t. the given recurrence equations. We will show that the process can be performed fully algorithmically by using appropriate tools. In the end we have extended the power of COSTA by allowing solving more and more general recurrences, specifically those admitting a polynomial upper bound on the height of the evaluation trees.

The plan of the paper is as follows: After this introduction, in Sec. 2 we summarise the main components of the COSTA-PUBS system and the process it follows for recurrence solving; in Sec. 3 we explain the main steps of our inference process by using a small running example; sections 4,5 , and 6 contains the technical details of respectively what do we mean by evaluating a non-deterministic recurrence, how to choose appropriate test points, and how to prove the obtained polynomial correct; finally, Sec. 7 surveys the related work and draws some conclusions.

\section{A Broad Overview of COSTA and PUBS}

The COSTA System is based on the classical approach to resource analysis, due to Wegbreit [25]. Given a Java bytecode program and a cost model, COSTA generates, in a first phase, a set of equations specifying the cost of the program as a function on the size of its input. COSTA provides several notions of size which depend on the input's type: The size of an integer is its value, whereas the size of an array is its number of elements. Finally, the size of an arbitrary object is the longest reachable pointer path that stems from that object. As an 
example, we show below a code fragment with a recurrence relation specifying its memory consumption:

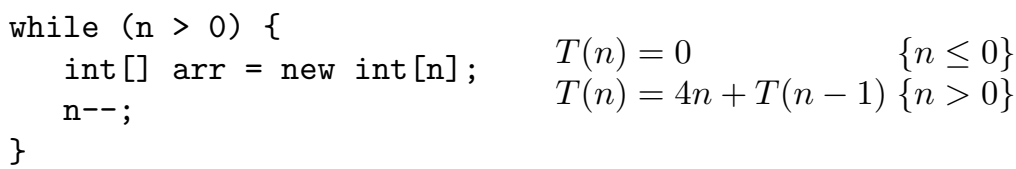

Although a recurrence relation captures precisely the cost of a program, an equivalent expression without recursion (closed form) gives a more intuitive idea about these costs from the programmer's point-of-view. For instance, the recurrence shown above admits the following closed-form: $T(n)=2 n^{2}+2 n$. So, the second phase of COSTA consists of the computation of a closed form for the previously generated set of equations. In contrast to already existing tools for solving recurrence relations [5], COSTA provides its own recurrence solver, PUBS [2]. The main reason is that, in practice, the set of equations capturing the cost of a program are not recurrence relations, but belong to the broader class of Cost Relation Systems (CRS), which are defined as follows:

Definition 1 (Adapted from [2]). A cost relation system is a finite set of mutually recursive equations of the form:

$$
T(\bar{x})=\exp +\sum_{j=1}^{l} D_{j}\left(\bar{y}_{j}\right) \quad \psi
$$

where $l \geq 0$, exp is a basic cost expression and $\psi$ is a set of linear relations on $\bar{x} \cup\left\{\bar{y}_{j}\right\}_{j=1 . . l} \cup \operatorname{vars}(\exp )$ specifying the conditions under which the equation can be applied.

The definition of basic cost expressions is not relevant to this paper, and it shall be omitted here (see [2] for details). As pointed out by Albert et al. it is possible to unfold the definitions of the $D_{j}$ symbols in order to obtain equations of the form $T(\bar{x})=\exp +\sum_{j=1}^{m} T\left(\bar{y}_{j}\right)$ for some $m \geq 0$. In the next sections we assume that this transformation has been done. The main difference between CRS and regular recurrence relations is non-determinism: when evaluating $T(\bar{v})$ for some concrete values, we might be able to apply more than one equation. Moreover, the arguments $T\left(\bar{y}_{j}\right)$ occurring in the recursive calls of an equation may not be uniquely determined by the guards, as in, for example, $T(x)=$ $1+T\left(x^{\prime}\right) \quad\left\{0 \leq x^{\prime}<x\right\}$. Thus the evaluation of $T(\bar{v})$ may give rise to different results and hence a CRS defines a relation instead of a function.

The PUBS approach for computing a closed form upper-bound is based on the notion of an evaluation tree (ET), which is the structure that arises from evaluating $T(\bar{v})$ with a concrete vector value $\bar{v}$. Each node in the ET contains the cost of its basic cost expression exp applied to the $\bar{v}$, and its children correspond to the recursive calls $T\left(\bar{v}_{j}\right)$, where $j \in\{1 . . m\}$. Notice that the evaluation of $T(\bar{v})$ may give place to several ETs, because of non-determinism. The computation of an upper bound to $T(\bar{x})$ amounts to finding upper-bounds to: 
- The number of base and recursive nodes of every possible ET, respectively denoted by $\operatorname{nb}(\bar{x})$ and $\operatorname{nr}(\bar{x})$.

- The value of the basic cost expressions occurring in the base and recursive nodes of every possible ET, respectively denoted by $\operatorname{cb}(\bar{x})$ and $\operatorname{cr}(\bar{x})$.

Given these, an upper bound $T^{+}(\bar{x})$ is computed as follows:

$$
T^{+}(\bar{x})=\operatorname{nb}(\bar{x}) * \operatorname{cb}(\bar{x})+\operatorname{nr}(\bar{x}) * \operatorname{cr}(\bar{x})
$$

The computation of $\operatorname{cb}(\bar{x})$ and $\operatorname{cr}(\bar{x})$ is beyond the scope of this paper. With regard to the number of nodes in the ET ( $\mathrm{nb}$ and $\mathrm{nr}$ ), these functions can be approximated from the ET's branching factor $b$ and maximal height $h(\bar{x})$ as follows:

$$
\operatorname{nb}(\bar{x})=b^{h(\bar{x})} \quad \operatorname{nr}(\bar{x})= \begin{cases}h(\bar{x}) & \text { if } b=1 \\ \frac{b^{h(\bar{x})}-1}{b-1} & \text { if } b>1\end{cases}
$$

The $h$ function stands for the length of the maximal call chain (without including the base case) that stems from the root of the ET. For example, with the CRSs shown in (1) the only (and hence the longest) chain reachable from $T(n)$ is as follows:

$$
\underbrace{T(n) \rightarrow T(n-1) \rightarrow T(n-2) \rightarrow \ldots \rightarrow T(1)}_{h(n)=n} \rightarrow T(0)
$$

In order to compute $h(\bar{x})$, PUBS derives a ranking function for $T$ by applying Podelski and Rybalchenko's method [19]. Unfortunately, this method fails when the ranking function does not depend linearly on the arguments $\bar{x}$, as the following example shows:

Example 1. Let us assume the following loop:

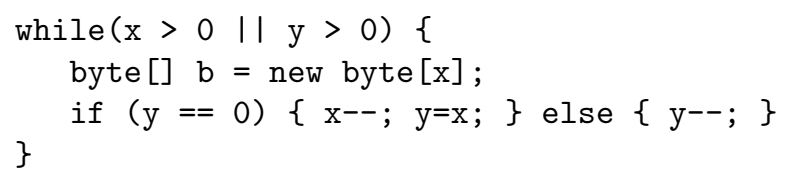

If we consider memory consumption, COSTA would obtain the following CRS:

$$
\begin{array}{ll}
T(x, y)=\operatorname{nat}(x) & \{x=0, y=0\} \\
T(x, y)=\operatorname{nat}(x)+T(x-1, x-1) & \{x>0, y=0\} \\
T(x, y)=\operatorname{nat}(x)+T(x, y-1) & \{x \geq 0, y>0\}
\end{array}
$$

where nat $(x)$ abbreviates $\max \{0, x\}$. This CRS cannot be solved by PUBS, since the length of the longest call chain depends on $(x, y)$ in a non-linear way.

$$
\underbrace{T(x, y) \rightarrow T(x, y-1) \rightarrow \ldots \rightarrow T(x, 0) \rightarrow T(x-1, x-1) \rightarrow \ldots \rightarrow T(0,0)}_{h(x, y)=\frac{1}{2} x^{2}+\frac{1}{2} x+y} \rightarrow T(0)
$$




\section{Interpolation-based call chain height analysis}

COSTA derives cost recurrence equations from Java bytecode. From these CRSs COSTA derives ranking functions. Ranking functions are used to bound the height of the evaluation trees. In COSTA such ranking functions are limited to linear expressions. It is our goal to improve the approximation of the height of the evaluation trees by considering general polynomial expressions. Using polynomial interpolation we aim to derive those polynomial expressions by analysing the call chain directly. To this purpose we first derive equations for the height of the call chain from the COSTA cost relation equations. Then, we derive upper bounds by polynomial interpolation.

Firstly, we must determine the function to be interpolated. We transform a given original CRS in order to obtain a recursive definition modeling the height $T_{h}(\bar{x})$ of the evaluation tree:

- for non-recursive equations of the form:

$$
T(\bar{x})=\exp \quad \psi \quad \text { we get: } \quad T_{h}(\bar{x})=0 \quad \psi
$$

- for recursive equations of the form:

$$
T(\bar{x})=\exp +\sum_{i=1}^{m} T\left(\overline{y_{i}}\right) \quad \psi \quad \text { we get: } \quad T_{h}(\bar{x})=1+\max _{i=1 \ldots m}\left\{T_{h}\left(\overline{y_{i}}\right)\right\} \quad \psi
$$

The function $T_{h}$ is multivalued: $T_{h}(\bar{x})$ is the collection of the lengths of all the call chains rooting in $\bar{x}$. Recall, that the function $h(\bar{x})$ is the maximum length of all possible call chains from $\bar{x}$, therefore $h(\bar{x})$ is the strict upper bound for $T_{h}(\bar{x})$.

Next, after we have the recurrence relations for $T_{h}(\bar{x})$, our aim is to find a polynomial $T_{h}^{+}(\bar{x})$ such that $T_{h}^{+}(\bar{x}) \geq h(\bar{x})$. If $h(\bar{x})$ is a polynomial, then $T_{h}^{+}(\bar{x})$ may be found by the standard interpolation. For instance, this is the case for the example 1. We will consider it in more detail later in this section. If $h(\bar{x})$ is not a polynomial then the standard interpolation must be adjusted. In both cases the idea is to generate a candidate for $T_{h}^{+}(\bar{x})$ based on its values in some finite collection of nodes. Then the candidate is checked. Roughly, checking is done by substituting it into the recurrence relation for $T_{h}(\bar{x})$.

In this paper we consider in more detail the issues related to the generating a candidate for an upper bound. The most important issue here is to find a collection of test nodes such that the corresponding interpolation problem have the solution (and it must be unique). There are many ways to obtain test nodes. In our work we use the gradient-based approach, which is described in Sec. 5.

Now we need to recapitulate some prerequisites from the classical interpolation theory that are necessary to understand the major challenge: finding test nodes. Recall that a polynomial of degree $d$ and dimension $s$ (the number of variables) has $N_{d}^{s}=\left(\begin{array}{c}d+s \\ s\end{array}\right)$ coefficients. Let a set of values $f_{i}$ of a real function $f$ be given. A set $W=\left\{\bar{w}_{i}: i=1, \ldots, N_{d}^{s}\right\}$ of points in a real $s$ dimensional space forms the set of interpolation nodes if there is a unique polynomial $p(\bar{z})=\Sigma_{0 \leq|j| \leq d} a_{j} \bar{z}^{j}$ with the total degree $d$ with the property $p\left(\bar{w}_{i}\right)=f_{i}$, 
where $1 \leq i \leq N_{d}^{s}$. In this case one says that the polynomial $p$ interpolates the function $f$ at the nodes $\bar{w}_{i}$. The polynomial interpolation exists and is unique under some conditions on the data, which are explored in polynomial interpolation theory [7]. For 1-variable interpolation this condition is well-known: all the test-nodes must be different. We have a closer look at 1-variable interpolation to provide the reader with the intuition behind the general case. A polynomial $p(z)$ of degree $d$ with coefficients $a_{1}, \ldots, a_{d+1}$ can be written as follows:

$$
a_{1}+a_{2} z+\ldots+a_{d+1} z^{d}=p(z)
$$

The values of the polynomial function in any pairwise different $d+1$ points determine a system of linear equations w.r.t. the polynomial coefficients. More specifically, given the set $\left(z_{i}, p\left(z_{i}\right)\right)$ of pairs of numbers, where $1 \leq i \leq d+1$, and coefficients $a_{1}, \ldots, a_{d+1}$, the set of equations can be represented in the following matrix form, where only the $a_{i}$ are unknown:

$$
\left(\begin{array}{ccccc}
1 & z_{1} & \cdots & z_{1}^{d-1} & z_{1}^{d} \\
1 & z_{2} & \cdots & z_{2}^{d-1} & z_{2}^{d} \\
\vdots & \vdots & \ddots & \vdots & \vdots \\
1 & z_{d} & \cdots & z_{d}^{d-1} & z_{d}^{d} \\
1 & z_{d+1} & \cdots & z_{d+1}^{d-1} & z_{d+1}^{d}
\end{array}\right)\left(\begin{array}{c}
a_{1} \\
a_{2} \\
\vdots \\
a_{d} \\
a_{d+1}
\end{array}\right)=\left(\begin{array}{c}
p\left(z_{1}\right) \\
p\left(z_{2}\right) \\
\vdots \\
p\left(z_{d}\right) \\
p\left(z_{d+1}\right)
\end{array}\right)
$$

The determinant of the left matrix, which contains the measurement points, is called the Vandermonde determinant. For pairwise different points $z_{1}, \ldots, z_{d+1}$ it is non-zero. This means that, as long as the output size is measured for $d+1$ different input sizes, there exists a unique solution for the system of equations and, thus, a unique interpolating polynomial.

The condition under which there exists a unique polynomial that interpolates multivariate data is not trivial. This condition on $W$ is geometrical: it describes a configuration, called Node Configuration A (NCA) [7], in which the points from $W$ should be placed in $\mathcal{R}^{s}$. The multivariate Vandermonde determinant computed from such points is non-zero. Thus, the corresponding system of linear equations w.r.t. the polynomial's coefficients has a unique solution. For a twodimensional polynomial of degree $d$, the condition on the nodes that guarantees a unique polynomial interpolation is as follows:

$N_{d}^{2}$ nodes forming a set $W \subset \mathcal{R}^{2}$ lie in a 2-dimensional $N C A$ if there exist lines $\gamma_{1}, \ldots, \gamma_{d+1}$ in the space $\mathcal{R}^{2}$, such that $d+1$ nodes of $W$ lie on $\gamma_{d+1}$ and $d$ nodes of $W$ lie on $\gamma_{d} \backslash \gamma_{d+1}, \ldots$, and finally 1 node of $W$ lies on $\gamma_{1} \backslash\left(\gamma_{2} \cup \ldots \cup \gamma_{d+1}\right)$. A simple example of NCA is given by a rectangular grid: there are $d+1$ parallel lines, such that some $d+1$ points lie on one line, $d$ points lie on another one, $d-1$ points belong on some third line, etc.

The NCA configuration for $s$ variables (s-dimensional space) is defined inductively on $s$ [7]. Let $\left\{\bar{z}_{1}, \ldots, \bar{z}_{N_{d}^{s}}\right\}$ be a set of distinct points in $\mathcal{R}^{s}$ such that there exist $d+1$ hyperplanes $K_{j}^{s}, 0 \leq j \leq d$ with

$$
\begin{aligned}
& \bar{z}_{N_{d-1}^{s}+1}, \ldots, \bar{z}_{N_{d}^{s}} \in K_{s}^{d} \\
& \bar{z}_{N_{j-1}^{s}+1}, \ldots, \bar{z}_{N_{j}^{s}} \in K_{j}^{s} \backslash\left\{K_{j+1}^{s} \cup \ldots \cup K_{d}^{s}\right\}, \text { for } 0 \leq j \leq d-1
\end{aligned}
$$


and each of set of points $\bar{z}_{N_{j-1}^{s}+1}, \ldots, \bar{z}_{N_{j}^{s}}, 0 \leq j \leq s$, considered as points in $\mathcal{R}^{s-1}$ satisfies NCA in $\mathcal{R}^{s-1}$.

\subsection{Application of the approach to an example}

Recall the recurrence relations in the example 1 . We have to find an interpolating polynomial of degree 2 for the corresponding call-tree-height function:

$$
\begin{array}{ll}
T_{h}(x, y)=0 & \{x=0, y=0\} \\
T_{h}(x, y)=1+T_{h}(x-1, x-1) & \{x \geq 1, y=0\} \\
T_{h}(x, y)=1+T_{h}(x, y-1) & \{x \geq 0, y \geq 1\}
\end{array}
$$

Evaluating $T_{h}$ in 6 points forming an NCA yields the following table:

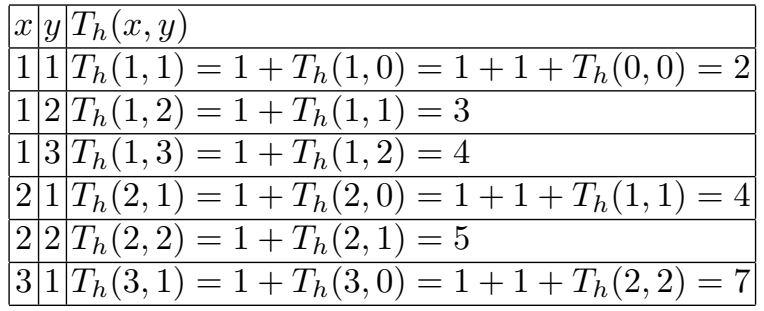

By solving the resulting system we get the polynomial $T_{h}^{+}(x, y)=\frac{1}{2} x^{2}+$ $\frac{1}{2} x+y$. It is a routine to check that it fits the recurrence relations. We compute the closed form expression for $T^{+}$by using (2):

$$
T^{+}(x, y)=\operatorname{nat}\left(\frac{1}{2} x^{2}+\frac{1}{2} x+y\right) * \operatorname{nat}(x)+\operatorname{nat}(x)
$$

and taking into account that the branching factor in this case is $b=1$.

\subsection{When standard interpolation needs adjustment}

When $h(\bar{x})$ is not a polynomial, but still, bounded by some polynomial, the standard interpolation must be adjusted. This is a topic of the ongoing research.

In particular, taking into account that $T_{h}^{+}(\bar{x}) \geq h(\bar{x})$, the interpolation problem may be generalised by adding variables $\delta_{i_{j}}$ to the system of linear equations for the coefficients of $T_{h}^{+}(\bar{x})$ so that the corresponding conditions in the nodes look like $T_{h}^{+}\left(\bar{x}_{i_{j}}\right)+\delta_{i_{j}}=h\left(\bar{x}_{i_{j}}\right)$. The amount of rows of the system should increase correspondingly, that is we need more test nodes. If by solving the system we obtain non-negative $\delta_{i_{j}}$, then we have obtained a good candidate for $T_{h}^{+}(\bar{x})$.

We study other possibilities to adjust standard interpolation for finding $T_{h}^{+}(\bar{x})$ such that $T_{h}^{+}(\bar{x}) \geq h(\bar{x})$, like considering in-equalities $T_{h}^{+}\left(\bar{x}_{i}\right) \geq h\left(\bar{x}_{i}\right)$ and minimising an appropriate objective function over the unknown coefficients of the polynomial $T_{h}^{+}(\bar{x})$. 


\subsection{Towards non-deterministic cost relation systems}

When the CRS defining $T_{h}$ is deterministic (as in our previous examples) it defines a single-valued function whose evaluation can be done in the usual way, e.g. by unfolding. If we want to compute a upper-bound to $h(\bar{x})$ we just choose a set of points in the domain of $T_{h}$ lying in a NCA configuration, and evaluate $T_{h}$ in these points, as shown in the examples above.

However, the computation of an upper-bound in non-deterministic CRSs is far more involved. The main reason is that we cannot just choose a point $\bar{x}$ and obtain all the possible results of the evaluation of $T_{h}(\bar{x})$, since there could be infinitely many ETs resulting from it. In the next two sections we will explain how to obtain testing nodes in which the value of $T_{h}$ is known, and how to perform a search on those nodes in which interpolation is more likely to result in a correct upper-bound (gradient-based approach).

\section{Evaluation of Cost Relation Systems}

Before dealing with the evaluation of a CRS we have to define its semantics. By the sake of simplicity, we consider only CRSs with a single recursive call (the extension to CRSs with several calls is straightforward):

$$
\begin{aligned}
& T_{h}(\bar{x})=0 \quad \psi_{b}(\bar{x}) \\
& T_{h}(\bar{x})=1+T_{h}\left(\bar{x}^{\prime}\right) \quad \psi_{r}\left(\bar{x}, \bar{x}^{\prime}\right)
\end{aligned}
$$

These equations define a relation $T_{h} \subseteq \mathbb{N}^{s} \times \mathbb{N}_{\infty}$, where $\mathbb{N}_{\infty} \stackrel{\text { def }}{=} \mathbb{N} \cup\{+\infty\}$. The pair $(\bar{x}, n)$ belongs to $T_{h}$ iff $n$ is a result of the evaluation of $T_{h}(\bar{x})$. The intuitive meaning of $(\bar{x},+\infty)$ being in $T_{h}$ is that the evaluation of $T_{h}(\bar{x})$ may lead to an infinite call chain. The ordering $\leq$ on natural numbers and the + operator is extended to $\mathbb{N}_{\infty}$ as usual. Given these conventions, the following definition provides a way to characterize the relation $T_{h}$ from the set of equations in (3).

Definition 2. The relation $T_{h}$ defined by the CRS in (3) is the greatest fixed point of the function $F: \mathcal{P}\left(\mathbb{N}^{s} \times \mathbb{N}_{\infty}\right) \rightarrow \mathcal{P}\left(\mathbb{N}^{s} \times \mathbb{N}_{\infty}\right)$, defined as follows:

$F(X)=\left\{(\bar{x}, 0) \mid \psi_{b}(\bar{x})\right\} \cup\left\{(\bar{x}, n+1) \mid \psi_{r}\left(\bar{x}, \bar{x}^{\prime}\right) \wedge\left(\bar{x}^{\prime}, n\right) \in X\right.$ for some $\left.\bar{x}^{\prime} \in \mathbb{N}^{s}, n \in \mathbb{N}_{\infty}\right\}$

We write $T_{h}=g f p F$.

As a notational convention, we consider relations $T_{h} \subseteq \mathbb{N}^{s} \times \mathbb{N}_{\infty}$ to be $m u l$ tivalued functions $T_{h}: \mathbb{N}^{s} \rightarrow \mathcal{P}\left(\mathbb{N}_{\infty}\right)$. Their domain, denoted by dom $T_{h}$, is the set of $\bar{x} \in \mathbb{N}^{s}$ such that $T_{h}(\bar{x}) \neq \emptyset$.

Example 2. The following CRS

$$
\begin{array}{ll}
T_{h}(x)=0 & \{x=0\} \\
T_{h}(x)=1+T_{h}\left(x^{\prime}\right) & \left\{x>0 \wedge x^{\prime}<x\right\}
\end{array}
$$

defines the following relation

$$
T_{h}=[0 \mapsto\{0\}, 1 \mapsto\{1\}, 2 \mapsto\{1,2\}, \ldots, i \mapsto\{1 . . i\}, \ldots]
$$


Example 3. Consider the following example:

$$
\begin{array}{ll}
T_{h}(x)=0 & \{x=0\} \\
T_{h}(x)=1+T_{h}\left(x^{\prime}\right) & \left\{x^{\prime}>x\right\}
\end{array}
$$

Let us prove that this CRS defines the following relation:

$$
T_{h}=\{(0,0),(0,+\infty),(1,+\infty),(2,+\infty), \ldots\}
$$

The operator $F$, applied to this particular case, is defined as follows:

$$
F(X)=\{(0,0)\} \cup\left\{(x, n+1) \mid x<x^{\prime} \wedge\left(x^{\prime}, n\right) \in X \text { for some } x^{\prime} \in \mathbb{N}, n \in \mathbb{N}_{\infty}\right\}
$$

It is easy to see that $F\left(T_{h}\right)=T_{h}$. Hence, $T_{h}$ is a fixed point. Now we prove that is the greatest one by contradiction: assume that there exists a $T_{h}^{\prime} \supset T_{h}$ such that $T_{h}^{\prime}=F\left(T_{h}^{\prime}\right)$. Since $T_{h}^{\prime}$ strictly extends $T_{h}$, we have two possibilities:

- $(x, 0) \in T_{h}^{\prime}$ for some $x \neq 0$. This cannot happen, since $T_{h}^{\prime}=F\left(T_{h}^{\prime}\right)$ and the only tuple that $F$ can return with a 0 in its right-hand side is $(0,0)$.

- $(x, n) \in T_{h}^{\prime}$ for some $x \geq 0$ and some $n$ different from 0 and $+\infty$. Then, there must exist some $x_{1}>x$ such that $\left(x_{1}, n-1\right) \in T_{h}^{\prime}$, which leads to a contradiction if $n=1$, as we have seen in the previous point. If $n>1$, and since $\left(x_{1}, n-1\right) \in T_{h}^{\prime}$, there exists another $x_{2}>x_{1}$ such that $\left(x_{2}, n-2\right) \in T_{h}^{\prime}$ and we apply the same reasoning as before. Eventually we will reach a tuple $\left(x_{n}, 0\right) \in T_{h}^{\prime}$ for some $x_{n}>0$, leading to a contradiction.

Therefore, the set $T_{h}$ shown above is the relation defined by this CRS. This relation can be given as the following multivalued function:

$$
T_{h}(x)= \begin{cases}\{0,+\infty\} & \text { if } x=0 \\ \{+\infty\} & \text { otherwise }\end{cases}
$$

In order to apply the techniques explained in Sec. 3, it is necessary to choose a set of points and determine the maximum value returned by $T_{h}$ when applied to each of these points. However, in general, it may be difficult to compute $\max T_{h}(\bar{x})$ for an arbitrary $\bar{x}$, due to the non-determinism of CRSs. In particular, there may be a possibly infinite amount of vectors $\bar{x}^{\prime} \in \mathbb{N}^{s}$ satisfying the recursive guard $\psi_{r}\left(\bar{x}, \bar{x}^{\prime}\right)$, and hence being eligible to be passed as argument to the recursive call to $T_{h}$.

Example 4. Assume the following CRS:

$$
\begin{array}{ll}
T_{h}(x)=0 & \{x \geq 100\} \\
T_{h}(x)=1+T\left(x^{\prime}\right) & \left\{0 \leq x<100, x<x^{\prime}\right\}
\end{array}
$$

We get $T_{h}(0)=\{1 . .100\}$, but there are infinitely many ways of deriving $(0,1) \in$ $T_{h}$. In general, for any $x^{\prime} \geq 100$ we obtain $\left(x^{\prime}, 0\right) \in T_{h}$ and hence $(0,1) \in T_{h}$. 
Given these difficulties, we will consider the evaluation of $T_{h}$ in a bottom-up fashion: we start from the set of points $A_{0}$ such that the evaluation of $T_{h}$ returns $\{0\}$. These points are known because they satisfy the base guard, but not the recursive one. In the next step, we consider the set of points $A_{1}$ that satisfy the recursive guard, but the corresponding recursive call falls into a base case. In general, our aim is to find a hierarchy of sets $A_{0} \subseteq A_{1} \subseteq \ldots \subseteq A_{i}$, where each $A_{i}$ contains the values of $x$ such that the evaluation of $T_{h}(x)$ does not require more than $i$ steps.

Definition 3. Given a relation $T_{h}: \mathbb{N}^{s} \rightarrow \mathcal{P}(\mathbb{N})$ and $i \in \mathbb{N}$, we define the set $A_{i}$ as follows:

$$
A_{i}=\left\{\bar{x} \in \operatorname{dom} T_{h} \mid \max T_{h}(\bar{x}) \leq i\right\}
$$

Example 5. Back to our Example 2, we obtain $A_{i}=\{0 . . i\}$ for each $i \in \mathbb{N}$, whereas in Example 3 we get $A_{i}=\{0\}$ for each $i \in \mathbb{N}$.

Our next step is to find a characterization of these $A_{i}$ sets in terms of the guards occurring in the CRS. This characterization is given as a set of predicates $\varphi_{i}$, defined as follows:

Definition 4. Given the $C R S$ in (3) and for each $i \in \mathbb{N}$, we define the predicate $\varphi_{i}$ as follows:

$$
\begin{gathered}
\varphi_{0}(\bar{x}) \stackrel{\text { def }}{=} \psi_{b}(\bar{x}) \wedge \forall \bar{x}^{\prime} \cdot \neg \psi_{r}\left(\bar{x}, \bar{x}^{\prime}\right) \\
\varphi_{i}(\bar{x}) \stackrel{\text { def }}{=} \varphi_{0}(\bar{x}) \vee\left[\left(\exists \bar{x}^{\prime} \cdot \psi_{r}\left(\bar{x}, \bar{x}^{\prime}\right)\right) \wedge \forall \bar{x}^{\prime} \cdot\left(\psi_{r}\left(\bar{x}, \bar{x}^{\prime}\right) \Rightarrow \varphi_{i-1}\left(\bar{x}^{\prime}\right)\right)\right] \quad \text { where } i>0
\end{gathered}
$$

By using quantifier elimination methods we can express every $\varphi_{i}$ as a finite union of convex polyhedra. In [18] a survey of quantifier elimination techniques can be found. Most of these methods only apply to dense linear orders [26]. Cooper [9] developed a quantifier elimination procedure for Presburger arithmetic extended with a divisibility predicate. This is the method we are going to use in the subsequent examples. In our implementation we rely on a computer algebra system and its extension to a computer logic system $[12,10]$.

Example 6. We get the following predicates from the CRS given in Example 2:

$$
\begin{aligned}
& \varphi_{0}(x) \equiv x=0 \wedge \neg \exists x^{\prime} .\left(x>0 \wedge x^{\prime}<x\right) \\
& \equiv x=0 \wedge \neg(x>0) \\
& \equiv x=0 \\
& \varphi_{1}(x) \equiv x=0 \vee\left(x>0 \wedge \forall x^{\prime} \cdot\left[x>0 \wedge x^{\prime}<x \Rightarrow x^{\prime}=0\right]\right) \\
& \equiv x=0 \vee(x>0 \wedge \neg[x>0 \wedge 1<x \wedge 1 \neq 0]) \quad \text { \{quantifier elimination\} } \\
& \equiv x=0 \vee x=1 \\
& \varphi_{2}(x) \equiv \ldots \\
& \equiv x \geq 0 \wedge x \leq 2
\end{aligned}
$$

Now we prove that these predicates characterize the $A_{i}$ sets. Without imposing special conditions on the CRSs, we can only prove that the $\varphi_{i}$ predicates offer sufficient conditions for belonging to the $A_{i}$ sets. More formally, 
$\left\{\bar{x} \in \mathbb{N}^{s} \mid \varphi_{i}(\bar{x})\right\} \subseteq A_{i}$ for each $i \in \mathbb{N}$. Strict inclusion may hold, in particular, when there are elements in the domain such that the evaluation of $T_{h}$ gets stuck, as the following example shows.

Example 7. Given the following CRS:

$$
\begin{array}{ll}
T_{h}(x)=0 & \{x=0\} \\
T_{h}(x)=1+T_{h}\left(x^{\prime}\right) & \left\{x \geq 2 \wedge\left(x^{\prime}=0 \vee x^{\prime}=1\right)\right\}
\end{array}
$$

We get $T_{h}=[0 \mapsto\{0\}, 1 \mapsto \emptyset] \cup[i \mapsto\{1\} \mid i \geq 2]$ and hence, $A_{0}=\{0\}$ and $A_{i}=\{0,2,3,4, \ldots\}$ for each $i \geq 1$. However, by applying the corresponding definition, we obtain $\varphi_{i} \equiv x=0$ for each $i \in \mathbb{N}$. This is an exact approximation to $A_{i}$ only when $i=0$.

We can ensure that the $\varphi$ predicates actually characterise the $A_{i}$ by imposing some mild conditions on our CRSs, namely, that every vector $\bar{x}$ satisfies at least one of the guards in the CRS.

Theorem 1. Given the CRS in (3), assume that $\psi_{b}(\bar{x}) \vee \exists \bar{x}^{\prime} \cdot \psi_{r}\left(\bar{x}, \bar{x}^{\prime}\right)$ holds for every $\bar{x} \in \mathbb{N}^{s}$. If $T_{h}: \mathbb{N}^{s} \rightarrow \mathcal{P}\left(\mathbb{N}_{\infty}\right)$ is the relation defined by this $C R S$, the following holds for each $i \in \mathbb{N}$ :

$$
A_{i}=\left\{\bar{x} \in \mathbb{N}^{s} \mid \varphi_{i}(\bar{x})\right\}
$$

Proof. By induction on $i$. The condition $\psi_{b}(\bar{x}) \vee \exists \bar{x}^{\prime} \cdot \psi_{r}\left(\bar{x}, \bar{x}^{\prime}\right)$ is only needed for proving the $\subseteq$ inclusion. The $\supseteq$ inclusion holds without special provisions.

\section{Searching for Test Nodes: Gradient-based Method}

Recall that $s$ is the dimension of the problem, that is the amount of variables on which $h$ depends. The sets $A_{i}$ correspond to predicates $\varphi_{i}(\bar{x})$ over variables $\bar{x}=\left(x_{1}, \ldots x_{s}\right)$. The graph of $h(\bar{x})$ in $s+1$-dimensional space is presented via the collection of sets $A_{i}^{\prime}:=\left(A_{i}, i\right)=\left\{(\bar{x}, i) \mid \bar{x} \in A_{i}\right\}$, which, informally speaking, form upside-down terraces. This surface is explained by the fact that $A_{i} \subseteq A_{i+1}$. An upper bound on $h$ "covers" the graph of $h$. Intuitively, the monotonicity behavior of a good upper bound and the monotonicity behavior of $h$ coincide, in the sense that the gradient of the bound at a point on the edge $A_{i}$ is the almost the same as the "gradient" of $h$ at this point. The gradient of a smooth scalar function $f(\bar{x})$ at a point $\bar{x}$ shows the direction of the greatest rate of increase of the function. It is defined as the vector of the derivatives: $\nabla(f):=$ $\left(\frac{\delta f}{\delta x_{1}}, \ldots, \frac{\delta f}{\delta x_{s}}\right)$. The graph of $h$ is not smooth, therefore here the notion of the gradient at the point $\bar{x} \in A_{i}$ is intuitive and taken as direction to the closest point on the next-level terrace $B_{i+1}:=A_{i+1} \backslash A_{i}$. The gradient-based method of finding test nodes mimics climbing up from $A_{i}^{\prime}$ to $A_{i+1}^{\prime}$. Based on this intuition, we propose the following procedure of finding test nodes.

\section{Inputs:}


- the amount of levels $l$, and sets $A_{i}, 0 \leq i \leq l$.

- the degree $d$ of a polynomial upper bound.

- the amount of climbing routes $r$ such that $r \times l \geq N(s, d)$, where $N(s, d)$ is the amount of coefficients of the polynomial in $s$ variables of the degree $d$.

- the initial terrace $A_{i_{0}}$. In the simplest case $i_{0}=0$.

- some $r$ points $\bar{x}_{i_{0} j}$ on the initial terrace, where $j$ is the route counter. If the set $A_{i_{0}}$ is linear then its vertices may be chosen as initial points.

2. Take $i:=i_{0}+1$, where $i$ is the level counter.

3. Take $j:=1$.

4. The next point $\bar{x}_{i j}$ on the $j$-th route is computed as the closest to $\bar{x}_{i-1 j}$ point on $B_{i}:=A_{i} \backslash A_{i-1}$ :

$$
\begin{aligned}
& \bar{x}_{i j}:=\operatorname{argmin}_{\bar{y}} \rho\left(\bar{x}_{i-1 j}, \bar{y}\right) \\
& \bar{y} \in B_{i} .
\end{aligned}
$$

5. Repeat the procedure for all $j=1, \ldots, r$.

6 . Repeat the procedure for all $i=i_{0}+1, \ldots, l$.

7. Choose from the obtained nodes a subset that satisfies NCA condition.

8. Solve the corresponding linear system for the coefficients of a candidate $T_{h}^{+}(\bar{x})$.

9. Output: The candidate $T_{h}^{+}(\bar{x})$.

Now, let's see how this procedure works for the recurrence for our example 1. Calculations have been implemented in a script for computer algebra reduce [12]. In principle, given a CRS, similar reduce-script may be generated automatically.

1. Inputs:

$-l:=7$ and the sets $A_{i}$ are generated by their definition using quantifierelimination procedure implemented in the reduce-package redlog [10]:

$$
\begin{aligned}
& A_{0} \text { is } x=0 \wedge y=0 \\
& A_{1} \text { is } x=0 \wedge y=0 \vee x-1=0 \wedge y=0 \vee x=0 \wedge y-1=0 \\
& A_{2} \text { is } A_{1} \vee x-1=0 \wedge y-1=0 \vee x=0 \wedge y-2=0 \\
& A_{3} \text { is } A_{2} \vee x-1=0 \wedge y-2=0 \vee x-2=0 \wedge y=0 \vee x=0 \wedge y-3=0 \\
& A_{4} \text { is } A_{3} \vee x-1=0 \wedge y-3=0 \vee x-2=0 \wedge y-1=0 \vee x=0 \wedge y-4=0 \\
& A_{5} \text { is } A_{4} \vee x-1=0 \wedge y-4=0 \vee x-2=0 \wedge y-2=0 \vee x=0 \wedge y-5=0 \\
& A_{6} \text { is } A_{5} \vee x-1=0 \wedge y-5=0 \vee x-2=0 \wedge y-3=0 \vee x-3=0 \wedge y=0 \\
& \vee x=0 \wedge y-6=0 \\
& A_{7} \text { is } \begin{array}{l}
A_{6} \vee x-1=0 \wedge y-6=0 \vee x-2=0 \wedge y-4=0 \vee x-3=0 \wedge y-1=0 \\
\vee x=0 \wedge y-7=0
\end{array} \\
& -d:=2 \text {, } \\
& -r:=3 \text {, } \\
& \text { - the initial level is } i_{0}:=3 \text {. } \\
& \text { - the initial points are the vertices of } A_{3}:(1,2),(2,0),(0,3) \text {. }
\end{aligned}
$$

2. The corresponding generated routes are (with levels from 4 to $7 \mathrm{incl}$.):

$$
\begin{aligned}
& \text { 1-st: }(1,3),(1,4),(1,5),(1,6) \\
& \text { 2-nd: }(2,1),(2,2),(2,3),(2,4) \\
& \text { 3-rd: }(0,4),(0,5),(0,6),(0,7)
\end{aligned}
$$


3. From these routes we pick up the following nodes: $(1,3)$ on level $4,(2,1)$ on level 4 and $(2,2)$ on level 5 , and $(0,4),(0,5),(0,6)$ on levels $4,5,6$ respectively.

4. The corresponding linear system is given by the interpolation conditions $T_{h}^{+}(1,3)=4, T_{h}^{+}(2,1)=4, T_{h}^{+}(2,2)=5, T_{h}^{+}(0,4)=4, T_{h}^{+}(0,5)=5$, $T_{h}^{+}(0,6)=6$. E.g., the first condition gives the first equation $a_{20}+9 a_{02}+$ $3 a_{11}+a_{10}+3 a_{01}+a_{00}=4$. The solution of the system is $a_{20}=a_{10}=\frac{1}{2}$, $a_{01}=1$ and the remaining coefficients are all zeros.

5. Output: The candidate $T_{h}^{+}(\bar{x})=\frac{x^{2}}{2}+\frac{x}{2}+y$. It passes the checking shown in Sec. 6.

The obtained routes and the graph of $T_{h}^{+}(x, y)$ are given in Fig. 1 .

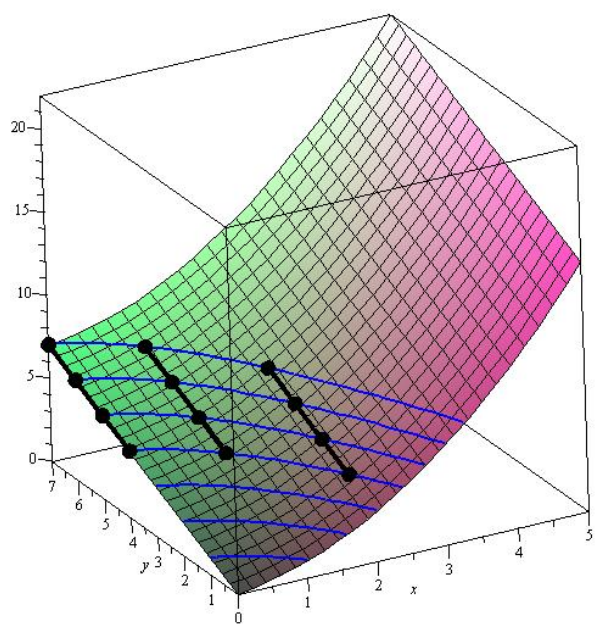

Fig. 1. The obtained 3 routes and the graph of $T_{h}^{+}(\bar{x})=\frac{x^{2}}{2}+\frac{x}{2}+y$ that in this case coincides with $h(x, y)$.

\section{Proving the Bound Correct}

A sufficient condition under which the upper bound $T_{h}^{+}(\bar{x})$ obtained by interpolation is an upper bound to the height $h(\bar{x})$ of the evaluation trees is given by the conjunction of:

$$
\begin{aligned}
& \forall \bar{x} \cdot T_{h}^{+}(\bar{x}) \geq 0 \\
& \forall \bar{x}, \bar{x}^{\prime} \cdot \psi_{r}\left(\bar{x}, \bar{x}^{\prime}\right) \Rightarrow\left(T_{h}^{+}(\bar{x}) \geq 1+T_{h}^{+}\left(\bar{x}^{\prime}\right)\right)
\end{aligned}
$$


Assuming that $\forall \bar{x} \cdot h(\bar{x}) \neq \infty$ (otherwise, there would be no bound for $h(\bar{x})$ ), the proof is a straightforward induction on $h(\bar{x})$.

But by considering the variables $\bar{x}, \bar{x}^{\prime}$ as real numbers, by knowing that $\psi_{r}\left(\bar{x}, \bar{x}^{\prime}\right)$ is a conjunction of linear inequalities, and that $T_{h}^{+}$is a multivariate polynomial of arbitrary degree, these formulas are decidable in Tarski's theory of real closed fields [24]. There are a number of tools available implementing improved versions of Tarski's procedure. For instance, QEPCAD [6] is free and offers an up-to-date version of Collins' algorithm [8]. If the formulas hold for real numbers, they will also hold for natural ones.

Should this check fail, this could indicate that the degree chosen for the inferred polynomial is not enough. If the degree $d$ is chosen very high at the beginning, this would result in needing many test points, and so in more computation time. But the polynomial inferred could be of a lower degree than $d$ because the coefficients of higher degree terms would be equal to 0 . A possible strategy is to start with a low degree such as $d=2$, and then increase $d$ by 1 or 2 at each iteration until either a degree succeeds or some time-out expires. In the latter case, we would report a fail to infer the bound.

\section{Conclusions and Related Work}

In this paper we have applied polynomial interpolation-based techniques in order to extend the PUBS recurrence solver, so that it can deal with a broader set of CRSs.

\section{Related Work}

We have taken the work described in [2] as our point of reference. In a more recent work [4] the authors improve the precision of PUBS by considering worstand best-case bounds to the cost of each loop iteration. The ideas described in this paper are orthogonal to those in [4] and can also be applied there.

In a different direction, COSTA has improved its memory analysis in order to take different models of garbage collection into account [3]. However, the authors claim that this extension does not require any changes to the recurrence solver PUBS. Thus, the techniques presented here should also fit with this extension.

In the field of functional languages, a seminal paper on static inference of memory bounds is [15]. A special type inference algorithm generates a set of linear constraints which, if satisfiable, they build a safe linear bound on the heap consumption.

One of the authors extended this type system in [13] in order to infer polynomial bounds. Surprisingly, the constraints resulting from the new type system are still linear ones. Although not every polynomial can be inferred by this system, the work was a remarkable step forward in the area. The language used is still functional, first-order and eager, but the resource inferred is a parameter. It could be either memory or time depending on some constants attached to the typing rules. A limitation of this work is that the inferred polynomials, even if 
they are multivariate ones, must not have multivariate terms. This limitation is removed in a more recent work [14].

The application of polynomial interpolation techniques makes it possible to derive polynomial complexity without any restriction in advance on the kind of polynomials. With interpolation polynomials can be multivariate and nonmonotonic. For size analysis of functional languages several interpolation results have been developed in the AHA Project [11]. First, a size analysis type system is developed together with language constraints such that sized type checking can be shown to be decidable. With polynomial interpolation type inference is made possible [16]. The full sized type system is given in [20]. In [23] it is shown how the basic type system, which is defined for list structures only, can be extended to allow algebraic data types. The size analysis systems give precise size functions. It has been shown that also general polynomial lower and upper bounds can be derived using polynomial interpolation [21].

Polynomial interpolation has also been applied to non-functional languages. For Java an analysis was made to derive ranking functions for loops [22].

\section{References}

1. Albert, E., Arenas, P., Genaim, S., Puebla, G., Zanardini, D.: COSTA: Design and Implementation of a Cost and Termination Analyzer for Java Bytecode. In: Formal Methods for Components and Objects (FMCO'07). pp. 113-133. LNCS 5382, Springer (October 2008)

2. Albert, E., Arenas, P., Genaim, S., Puebla, G.: Closed-form upper bounds in static cost analysis. J. Autom. Reasoning 46(2), 161-203 (2011)

3. Albert, E., Genaim, S., Gómez-Zamalloa, M.: Parametric inference of memory requirements for garbage collected languages. In: Vitek, J., Lea, D. (eds.) ISMM. pp. 121-130. ACM (2010)

4. Albert, E., Genaim, S., Masud, A.N.: More precise yet widely applicable cost analysis. In: Jhala, R., Schmidt, D.A. (eds.) VMCAI. Lecture Notes in Computer Science, vol. 6538, pp. 38-53. Springer (2011)

5. Bagnara, R., Zaccagnini, A., Zolo, T.: The automatic solution of recurrence relations. I. Linear recurrences of finite order with constant coefficients. Quaderno 334, Dipartimento di Matematica, Università di Parma, Italy (2003), available at http://www.cs.unipr.it/Publications/

6. Brown, C.W.: QEPCAD: Quantifier Elimination by Partial Cylindrical Algebraic Decomposition. http://www.cs.usna.edu/qepcad/B/QEPCAD.html (2004)

7. Chui, C.K., Lai, M.J.: Vandermonde determinants and lagrange interpolation in $R^{s}$. In: Nonlinear and Convex Analysis, Proceedings in Honor of Ky Fan. pp. 23-35. Marcel Dekker Inc., N.Y. (1987)

8. Collins, G.E.: Quantifier Elimination for Real Closed Fields by Cylindrical Algebraic Decomposition. In: Barkhage, H. (ed.) Automata Theory and Formal Languages. LNCS, vol. 33, pp. 134-183. Springer (1975)

9. Cooper, D.C.: Theorem proving in arithmetic without multiplication. Machine Inteligence 7, 91-100 (1972)

10. Dolzmann, A., Sturm, T.: Redlog user manual. Tech. Rep. MIP-9905, FMI, Universität Passau (1999), edition 2.0 for Version 2.0 
11. van Eekelen, M., Shkaravska, O., van Kesteren, R., Jacobs, B., Poll, E., Smetsers, S.: AHA: Amortized Heap space usage Analysis. In: Morazán, M. (ed.) Selected revised papers of the 8th international symposium on Trends in Functional Programming (TFP'07). pp. 36-53. Intellect, New York, USA (2007)

12. Hearn, A.C.: REDUCE. User's Manual. Version 3.8 (2004)

13. Hoffmann, J., Hofmann, M.: Amortized Resource Analysis with Polynomial Potential. A Static Inference of Polynomial Bounds for Functional Programs. In: ESOP 2010, LNCS 6012. pp. 287-306. Springer (2010)

14. Hoffmann, J., Aehlig, K., Hofmann, M.: Multivariate amortized resource analysis. In: Ball, T., Sagiv, M. (eds.) POPL. pp. 357-370. ACM (2011)

15. Hofmann, M., Jost, S.: Static prediction of heap space usage for first-order functional programs. In: Proc. 30th ACM Symp. on Principles of Programming Languages, POPL'03. pp. 185-197. ACM Press (2003)

16. van Kesteren, R., Shkaravska, O., van Eekelen, M.: Inferring static nonmonotonically sized types through testing. In: Proceedings of 16 th international workshop on Functional and (Constraint) Logic Programming (WFLP'07). Electronic Notes in Theoretical Computer Science, vol. 216C, pp. 45-63. Paris, France (2007)

17. Lucas, S.: Polynomials over the reals in proofs of termination: from theory to practice. RAIRO Theoretical Informatics and Applications 39(3), 547-586 (2005)

18. Nipkow, T.: Linear quantifier elimination. J. Autom. Reasoning 45(2), 189-212 (2010)

19. Podelski, A., Rybalchenko, A.: A complete method for the synthesis of linear ranking functions. In: Steffen, B., Levi, G. (eds.) VMCAI. Lecture Notes in Computer Science, vol. 2937, pp. 239-251. Springer (2004)

20. Shkaravska, O., van Eekelen, M., van Kesteren, R.: Polynomial size analysis of first-order shapely functions. Logical Methods in Computer Science 5(2:10), 1-35 (2009), selected Papers from TLCA 2007

21. Shkaravska, O., van Eekelen, M., Tamalet, A.: Collected size semantics for functional programs over lists. In: Scholz, S.B. (ed.) Revised selected papers of the 20th international symposium on Implementation and Application of Functional Programming (IFL'08). LNCS, vol. 5836. Springer-Verlag, University of Hertfordshire, UK (2011), to appear.

22. Shkaravska, O., Kersten, R., van Eekelen, M.: Test-based inference of polynomial loop-bound functions. In: Proceedings of the 8th International Conference on the Principles and Practice of Programming in Java, PPPJ 2010. ACM (2010)

23. Tamalet, A., Shkaravska, O., van Eekelen, M.: Size analysis of algebraic data types. In: Achten, P., Koopman, P., Morazán, M.T. (eds.) Selected revised papers of the 9th international symposium on Trends in Functional Programming (TFP'08). pp. 33-48. Intellect (2009)

24. Tarski, A.: A Decision Method for Elementary Algebra and Geometry. University of California Press, Berkeley (1948)

25. Wegbreit, B.: Mechanical program analysis. Commun. ACM 18(9), 528-539 (1975)

26. Weispfenning, V.: The complexity of linear problems in fields. J. Symb. Comput. $5(1 / 2), 3-27(1988)$ 